\title{
EVALUATION OF LIFE QUALITY IN PATIENTS WITH HAEMOPHILIA
}

\author{
Lavinia Hogea ${ }^{1}$, Laura Nussbaum ${ }^{1,2}$ \\ ${ }^{1}$ Neuroscience Department, "Victor Babes" University of Medicine and Pharmacy, Timisoara, Romania \\ ${ }^{2}$ Psychiatry and Neurology Pediatric Clinic, Timisoara, Romania
}

\begin{abstract}
There is an increasing concern for the evaluation of individual experience and perception of health condition by the cronic patients. World Health Organization Quality of Life (WHOQOL-100) is one of the most beneficial instruments for assessment of life quality in haemophilic patients.

The aim of the study is evaluation of life quality in haemophilic patients admitted in the Medical Center of Evaluation and Rehabilitation "Cristian Serban", Buzias, and Pediatric Clinic Children's Hospital "Luis Turcanu", Timisoara, Romania.
\end{abstract}

Keywords: haemophilia, quality of life

\section{INTRODUCTION}

Haemophilia, being a chronic, invalidating disease, encumbered by the permanent risk of bleeding puts its mark on the patients quality of life due to the many complications and sequelae produced by both the disease as well as by the treatment.

The numerous admissions, lack of employment, absences from school or work and financial problems due to deficient social insertion, have a negative impact on quality of life.

In this study were used the World Health Organization Quality of Life (WHOQOL-100) for measuring quality of life domains (general, physically, psychologically, independence, social relationships, the environment, spirituality) and anamnesis.

\section{MATERIAL AND METHOD}

The study included a number of 100 patients with $\mathrm{A}$ and $\mathrm{B}$ severe hemophilia with age between 16-45 years old, and the control group consists of 100 people without hemophilia. The control group consists of people without hemophilia, in various public institutions in Timisoara.
The data was collected by using the following questionnaires: World Health Organization Quality of Life (WHOQOL) were used. (Pearsons-r parameter)

\section{RESULTS}

Questions assessing the overall quality of life explore the way in which a person evaluates the overall quality of life, health and well-being. As expected, the overall quality of life and the health perception is deeply affected for the group of study patients compared to the group of clinically healthy subjects.

It is observed that there are significant statistical differences between the two groups in almost all areas of quality of life study (Table 1). Highly significant statistical differences being observed in the general domain of health, the environment, the psychological domain, social relationships and in the domain of spirituality/religion/personal beliefs ( $.001 ; \mathrm{p}<.05)$.

To investigate the differences between groups, and the effect of interaction regarding the quality of 
life, an univariate ANOVA has been realized 2 (people suffering from hemophilia, and people who do not suffer from hemophilia) X 3 (age 16-25 years old, 26-35 years old, 36-45 years old).

TABLE 1. $3 X 2$ factorial ANOVA summary table on the general quality of life

\begin{tabular}{|c|c|c|c|c|c|}
\hline $\begin{array}{c}\text { Source } \\
\text { dispersion }\end{array}$ & SS & Df & MS & $\boldsymbol{F}$ & $\boldsymbol{P}$ \\
\hline Lot & 237,959 & 1 & 237,959 & $31,099^{* *}$ & $.000^{* *}$ \\
Age & 30,368 & 2 & 15,184 & 1,984 & .140 \\
Lot X Age & 0,651 & 2 & 0,325 & 0,043 & .958 \\
Error & 1484,433 & 194 & 7,652 & & \\
Total & 40451,00 & 200 & & & \\
\hline
\end{tabular}

Note: ${ }^{* *} p<.001 ; * p<.05$

Of the three ratios $\mathrm{F}$, shown in Table 1, only one is statistically significant: $\mathrm{F}$ ' lot ' $\mathrm{F}(1,194)=$ $31,099, \mathrm{p}<.001(\mathrm{M}=12.33$ versus $\mathrm{M}=15.40)$. This means that there are statistically significant differences between non-hemophiliacs and hemophiliacs, hemophiliacs have a lower level on the general domain of quality of life, compared to non-hemophiliacs, due to the fact that the main effect for groups is statistically significant.

The purpose of assessing the general state of the quality of life is to highlight the effect that different pathological events have on the patient's life, taking into account the personal satisfaction and participation in social life.

The importance of subjective assessment of general condition of quality of life is recognized (1), thus proving that significant differences exist both between patients and healthy people, and between the one made by the physician and patient (2).

The low score within the domain of the overall quality of life, is because the subjects are self-assessing their overall health state as being precarious and, moreover, they consider that there is a possibility for their condition to worsen.

TABLE 2. 3X2 factorial ANOVA summary table on the physical quality of life

\begin{tabular}{|c|c|c|c|c|c|}
\hline $\begin{array}{c}\text { Source } \\
\text { dispersion }\end{array}$ & SS & Df & MS & $\boldsymbol{F}$ & $\boldsymbol{P}$ \\
\hline Lot & 4,890 & 1 & 4,890 & 2,046 & .15 \\
Age & 4,256 & 2 & 2,128 & 0,890 & .41 \\
Lot X Age & 10,955 & 2 & 5,478 & 2,292 & .10 \\
Error & 463,718 & 194 & 2,390 & & \\
Total & 28613,720 & 200 & & & \\
\hline
\end{tabular}

Note: ${ }^{* *} p<.01 ; * p<.05$

Regarding the physical domain (Table 2), the results show that there are no statistically significant differences between non-hemophiliac and hemophiliac: $\mathrm{F}(1,194)=2,046 \mathrm{p}>.05(\mathrm{M}=11.84$ versus $M=11.87)$.
One of the explanations is that for the questionnaire used there are no normative values for Romania, which is why the comparison has been made with a control group consisting of the same number of subjects with similar age. It is possible that the results may vary because, when calculating normative values, the group is much larger.

At the same time, the patients who were examined were at the time of the test in a physical rehabilitation clinic where they were closely monitored, a fact that may explain why this domain was unaffected.

TABLE 3. 3X2 factorial ANOVA summary table on the psychological quality of life

\begin{tabular}{|c|c|c|c|c|c|}
\hline $\begin{array}{c}\text { Source } \\
\text { dispersion }\end{array}$ & SS & Df & MS & $\boldsymbol{F}$ & $\boldsymbol{P}$ \\
\hline Lot & 27,642 & 1 & 27,642 & $17,713^{* *}$ & $.000^{* *}$ \\
Age & 6,975 & 2 & 3,487 & 2,235 & .110 \\
Lot X Age & 2,638 & 2 & 1,319 & 0,845 & .431 \\
Error & 302,737 & 194 & 1,560 & & \\
Total & 30428,600 & 200 & & & \\
\hline
\end{tabular}

Note: $* * p<.001 ; * p<.05$

Significant statistical results between the two groups: $\mathrm{F}$ ' ${ }^{\prime}$ lot ' $\mathrm{F}(1,194)=17,713 \mathrm{p}<.001(\mathrm{M}=$ 11.87 versus $\mathrm{M}=12.65)$ were obtained on the psychological domain (Table 3).

Although in recent years there has been many advances in the treatment of hemophilia, there are numerous problems that still remain unsolved, including debilitating disease complications and infancy lethality, spontaneous bleeding risk, social integration, the lack of a prophylactic treatment, fear of infection through blood products, frequent hospital admissions, they put their mark on the psychic status of patients.

Increased incidence of complications related to the disease and treatment, chronic nature of the disease, poor social integration and social relations are the consequences of the various psychological disturbances of hemophiliac patients.

A survey conducted on 83 children and adolescents with hemophilia A and B, aged between 5 and 19 years, using structural interviews from DSM-IV have noted the prevalence of major depressive disorder, a disorder of the separation anxiety disorder and suicidal behavior in children and adolescents with hemophilia. (3)

Physical field and independent field are closely related to each other, it was expected that the effect of the variable group field of the variable quality of life to be statistically insignificant: $F(1,194)=$ $0,176, p>.05(M=12.03$ versus $M=11.91)$ (Table 4$)$. 
TABLE 4. $3 X 2$ factorial ANOVA summary table on the independence of quality of life

\begin{tabular}{|c|c|c|c|c|c|}
\hline $\begin{array}{c}\text { Source } \\
\text { dispersion }\end{array}$ & SS & Df & MS & $\boldsymbol{F}$ & $\boldsymbol{P}$ \\
\hline Lot & 0,185 & 1 & 0,815 & 0,176 & .67 \\
Age & 4,519 & 2 & 2,259 & 2,147 & .12 \\
Lot X Age & 1,606 & 2 & 0,803 & 0,763 & .46 \\
Error & 204,185 & 194 & 1,052 & & \\
Total & 28896,080 & 200 & & & \\
\hline
\end{tabular}

Note: ${ }^{* *} p<.001 ; * p<.05$

TABLE 5. 3X2 factorial ANOVA summary table on the social relations of quality of life

\begin{tabular}{|c|c|c|c|c|c|}
\hline $\begin{array}{c}\text { Source } \\
\text { dispersion }\end{array}$ & SS & Df & MS & $\boldsymbol{F}$ & $\boldsymbol{P}$ \\
\hline Lot & 23,615 & 1 & 23,615 & $18,427^{* *}$ & $.000^{* *}$ \\
Age & 0,596 & 2 & 0,298 & 0,233 & .793 \\
Lot X Age & 7,507 & 2 & 3,754 & $2,929^{*}$ & .056 \\
Error & 248,625 & 194 & 1,282 & & \\
Total & 28277,068 & 200 & & & \\
& & & & & \\
\hline
\end{tabular}

Note: ${ }^{* *} p<.001 ; * p<.05$

A low score of social relations means frequent and marked interference with normal social activities due to physical or emotional problems.

Social factors play an especially important role in maintaining the health and hemophilia through its consequences affects the patient suffering from the disease not just physically but also socially. Therefore, a hemophiliac child needs the best treatment from infancy to prevent complications and to be able to be socially active.

The chronic nature of the disease, its evolution, affects not only the patient's life but also that of his family. For this reason the scores obtained reported significant differences between the two groups studied.

TABLE 6. 3X2 factorial ANOVA summary table on the environment

\begin{tabular}{|c|c|c|c|c|c|}
\hline $\begin{array}{c}\text { Source } \\
\text { dispersion }\end{array}$ & SS & Df & MS & $\boldsymbol{F}$ & $\boldsymbol{P}$ \\
\hline Lot & 48,349 & 1 & 48,349 & $36,159^{* *}$ & $.000^{* *}$ \\
Age & 14,683 & 2 & 7,342 & $5,491^{*}$ & $.005^{*}$ \\
Lot X Age & 1,092 & 2 & 0,546 & 0,408 & .665 \\
Error & 259,402 & 194 & 1,337 & & \\
Total & 28828,750 & 200 & & & \\
\hline
\end{tabular}

Note: $* * p<.001 ; * p<.01$

Subjects from the clinical group assess unfavorable the quality and access to health and social protection, which leads to significant statistical differences between the two groups.

As with other chronic conditions and in the case of hemophilia, appropriate treatment does not cure the disease, but maintains a normal way of life, free from suffering and stopping evolution towards deficiency, disability or handicap.

Of course, along with proper treatment, other important factors are also involved like those cultural, familial, socio-demographics, education and professional training, the economic level of the country, the accessibility to treatment, safety and physical security of everyone's ability to acquire new skills and to use them. (4)

Health in childhood has direct consequences for the social and economic status in adulthood. (5) So that one can speak of a social causation, meaning that a child who comes from a family with a low social-economic status will in turn become an adult with a low social-economic status. (6.7)

Poverty is one of the most important determinants of the disease, it is associated with an increased risk of morbidity, a low compliance to treatment, a lower life expectancy, but it is also the generator of numerous psychological manifestations, all with negative health consequences.

The absence of adequate therapeutic means, of centers specializing in the treatment of hemophilia where multidisciplinary teams exist, is responsible for the decrease in compliance to treatment and quality of life.

TABLE 7. $3 X 2$ factorial ANOVA summary table on the spiritual quality of life

\begin{tabular}{|c|c|c|c|c|c|}
\hline $\begin{array}{c}\text { Source } \\
\text { dispersion }\end{array}$ & SS & Df & MS & $\boldsymbol{F}$ & $\boldsymbol{P}$ \\
\hline Lot & 57,572 & 1 & 57,572 & $10,281^{*}$ & $.002^{*}$ \\
Age & 17,739 & 2 & 8,870 & 1,584 & .208 \\
Lot X Age & 60,582 & 2 & 30,291 & $5,409^{*}$ & $.005^{*}$ \\
Error & 1086,336 & 194 & 5,60 & & \\
Total & 32534,000 & 200 & & & \\
\hline
\end{tabular}

Note: $* * p<.001 ; * p<.05$

In Table 7 are found the statistically significant differences between the two groups in this field, the data being low regarding the subjects from the clinical group on the two age classes (16-25 years and $36-45$ years) and in the 26-35 years category, the scores are lower in the control group.

This area covers the personal beliefs related to quality of life. Their own beliefs may generate the state of well-being, can help the person to cope with the difficulties of life. Also personal beliefs and spirituality give feelings of safety, comfort, well-being, inner strength, as well as giving life a purpose.

\section{CONCLUSIONS}

We can thus conclude that there are statistically significant differences between non-hemophiliacs 
and hemophiliacs, hemophiliacs have a lower level of quality of life regarding the general, psychological, social relations, the environment and spirituality domain compared to non-hemophiliacs.

Similar results were obtained in other studies performed over the years, such as that of his Royal and his collaborators. (8) All the hemophiliac patients which have been studied had lower scores compared to the general population at the physical and overall health dimensions. Hemophiliacs who received prophylactic treatment had less affected scores at the dimension of the body pain, general health, physical function, mental health, social functioning.

In another study conducted in Italy by Trippoli and his collaborators (9), on a group of 56 hemophiliac patients, results have shown that the age of patients is an important factor for the quality of life.

Solovieva (10) has followed in a study, the quality of life, the clinical modifications, the intensity of pain and the functional disability, on 150 patients for a period of 3 years, and the results obtained showed that the impairment and musculoskeletal changes are predictors for physical impairment, pain, vitality and social integration. Other factors such as the bleeding frequency and the orthopedic surgeries, significantly affects the quality of life.

Using the tools EuroQol (EQ-5D) and The Short Form (36) Health Survey (SF-36) correlated to anamnesis data (age, orthopedic surgery, the number of bleedings per year, severity of disease, HIV infection), Miners and his collaborators (11), on a group of 249 hemophiliac patients, have noted that the severity of hemophilia is an important influence factor of life quality in comparison with the general population. And Mohlo and his collaborators (12) in the study that included a total number of 116 hemophiliac patients concluded that the most affected domains are the bodily pain, general health and vitality. In their study, quality of life is less affected in young patients due to the small degree of joint damage.

In a study conducted in Romania in which the WHOQL-100 tool was used on 100 patients that were over 15 years old, it has been noted that the scores of all the quality of life domains with the exception of spirituality were affected. (13)

\section{REFERENCES}

1. Slevin M.L., Who should measure quality of life, the doctor or the patients?, British Journal of Cancer, 1988, 57(1):109-112

2. Pearlman R.A., et al., Quality of life in chronic diseas: perception of elderly patients, Journal of Gerontology, 1988, 43(2): M25-30

3. Ghanizadeh A., Depression, anxiety and suicidal behaviour in children and adolescents with haemophilia, Haemophilia, 2009, 15(2):528-532

4. Luchtman-Jones L., et al., Consideration in the evaluation of haemophilia patients for short term prophylactic therapy, Haemophilia, 2006, 12(1):82-86

5. Szucs T.D., et al., Socioeconomic impact of haemophilia care: results of a pilot study, Haemophilia, 1996, 2(4):211-217

6. Lundberg 0 ., Childhood living condition, health status, and social mobility: a contribution to the health selection debate, European Sociological Review, 1991, 7(2):149-162

7. Wadsworth M.E.J., Serious illness in childhood and its association with later-life achievement. In Class and Health: Research and Longitudinal Data, 1986, 1-20

8. Royal S., et al., Quality of life differences between prophylactic and on-demand factor replacement therapy in European haemophilia patients, Haemophilia, 2002, 8(1): 44-50

9. Trippoli S., et al., Multivariate analysis of factors infuencing quality of life and utility in patients with haemophilia, Haematologica, 2001, 86:722-728

10. Solovieva S., Clinical severity of disease, functional disability and health related quality of life. Three-year follow-up study of 150 Finnish with coagulations disorders, Haemophilia, 2001, 7(1):53-63

11. Miners A., et al., Assessing health-related quality of life in individuals with haemophilia, Haemophilia, 1999, 5(6):378-385

12. Mohlo P., et al., Epidemiological survey of the orthopaedic status of severe haemophilia A and B patients in France, Haemophilia, 2000, 6(1):23-32

13. Şerban M., et al. Multicentric evaluation of health quality of life in Romanian haemophiliacs, 2009, 59. 